\title{
Lattice QCD and chiral mesons
}

\author{
Sergio Caracciolo ${ }^{\mathrm{a}}$, Fabrizio Palumbo ${ }^{\mathrm{b}}$, and Roberto Scimia $^{\mathrm{c}}$

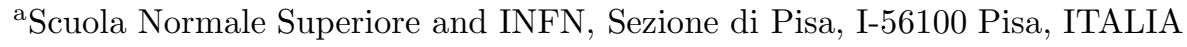

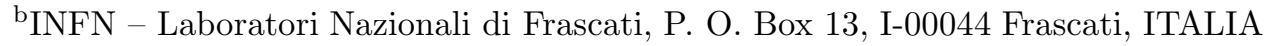 \\ ${ }^{\text {c}}$ Dip. di Fisica dell'Università and INFN, Sezione di Perugia, Via A. Pascoli, I-06100 Perugia, ITALIA
}

The standard QCD action is improved by the addition of irrelevant operators built with chiral composites. An effective Lagrangian is derived in terms of auxiliary fields, which has the form of the phenomenological chiral Lagrangians. Our improved QCD action appears promising for numerical simulations as the pion physics is explicitely accounted for by the auxiliary fields.

QCD in the high energy limit is efficiently described by the perturbative expansion in the gauge coupling constant. For the low energy properties instead, we do not have an equally satisfactory theory.

At the phenomenological level a description of the low energy physics related to the symmetry breaking of the chiral invariance is obtained by using the phenomenological chiral Lagrangians 佂, 21, but a direct derivation from the basic theory of quarks and gluons is still lacking.

At a more fundamental level the lattice formulation has allowed us to investigate the quark confinement [3] and the spontaneous breaking of chiral invariance, but due to the complexities related to the definition of chiral fermions [3, 4], in numerical simulations the chiral limit is achieved only through a fine-tuning procedure. Even though these difficulties can be overcome, we remain unable to unify the treatment of high energy and low energy properties.

For these reasons we have developed an approach based on the use of quark composites as fundamental variables. The idea behind it is that a significant part of the binding of the hadrons can be accounted for in this way, so that the "residual interaction" is sufficiently weak for a perturbative treatment.

The quark-composites approach is in principle fairly general, since it allows us to treat all the hadrons composite of quarks, but for technical reasons the composites with the quantum numbers of mesons and barions are treated in a different way. The nucleonic composites, for instance, naturally satisfy the Berezin integration rules and we derived the substitution rules which allow us to replace polynomials of the quark fields by appropriate polynomials of these composites [5] in the partition function. The mesonic composites instead, due to the complexity of the integral over even elements of a Grassmann algebra, are replaced by auxiliary fields [6] by means of the Stratonovich-Hubbard transformation [7]. Even though our effective action is not renormalizable by power counting, due to the renormalizability of QCD only a finite number of free parameters can be generated by the counterterms because of the BRS identities.

We assume the modified partition function

$Z=\int[d V][d \bar{\lambda} d \lambda] \exp \left[-S_{Y M}-S_{q}-S_{C}\right]$,

where $S_{Y M}$ is the Yang-Mills action, $S_{q}$ is the action of the quark field and $S_{C}$ is a four fermions irrelevant operator which provides the kinetic terms for the quark composites with the quantum numbers of the chiral mesons. $\lambda$ is the quark field while the gluon field is associated to the link variables $V$. Differentials in square brackets are understood to be the product of the differentials over the lattice sites and the internal indices. All the fields live in an euclidian lattice of spacing $a$. We introduce the following notation for the sum 
over the lattice

$(f, g)=a^{4} \sum_{x} f(x) g(x)$.

In this notation the quark action is

$S_{Q}=(\bar{\lambda}, Q \lambda)$.

We do the Wilson choice for the quark wave operator

$Q=Q_{D}-Q_{W}, Q_{D}=\gamma_{\mu} \bar{\nabla}_{\mu}, \quad Q_{W}=a \frac{r}{2} \square$.

The symmetric derivative $\bar{\nabla}_{\mu}$ and the Laplacian $\square$ are covariant and are defined in terms of the right/left derivatives

$\left(\nabla_{\mu}^{ \pm}\right)_{x y}= \pm \frac{1}{a}\left(\delta_{x \pm \hat{\mu}, y} V_{ \pm \mu}(x)-\delta_{x y}\right)$

according to

$\bar{\nabla}_{\mu}=\frac{1}{2}\left(\nabla_{\mu}^{+}+\nabla_{\mu}^{-}\right), \quad \square=\sum_{\mu} \nabla_{\mu}^{+} \nabla_{\mu}^{-}$

with standard conventions for the link variables.

The chiral composites are the pions and the sigma

$\overrightarrow{\hat{\pi}}=i a^{2} k_{\pi} \bar{\lambda} \gamma_{5} \vec{\tau} \lambda, \quad \hat{\sigma}=a^{2} k_{\pi} \bar{\lambda} \lambda$.

$\gamma_{5}$ is assumed hermitian, the $\vec{\tau}$ 's are the Pauli matrices and a factor of dimension (length) ${ }^{2}$, necessary to give the composites the dimension of a scalar, has been written in the form $a^{2} k_{\pi}$ for convenience (see below).

Since for massless quarks the QCD action is chirally invariant, the action of the chiral mesons must be, apart from a linear breaking term, $O(4)$ invariant. It must then have the form

$S_{C}=\frac{1}{4}\left\langle\left(\hat{\Sigma}^{\dagger}, C \hat{\Sigma}\right)\right\rangle-\frac{1}{4}\left\langle\left(\hat{\Sigma}^{\dagger}+\hat{\Sigma}\right) m_{q}\right\rangle$,

where

$\hat{\Sigma}=\hat{\sigma}+i \vec{\tau} \cdot \overrightarrow{\hat{\pi}} \gamma_{5}, \quad\langle A\rangle=\operatorname{tr}^{i \text { sospin }} A$.

There is a long history of 4-fermions interactions and their relation to QCD, starting from the Nambu-Jona-Lasinio [8] and Gross-Neveu [9] models, until the so-called chirally extended QCD or $\chi \mathrm{QCD}$ (see also for example [10]).
Our demand of irrelevance of $S_{C}$, needed to maintain the renormalization properties of $\mathrm{QCD}$, and heuristic considerations, based on experience with simple, solvable models, lead [6] to the following form of the wave operator of the chiral composites

$C=-\frac{\rho^{4}}{a^{4}} \frac{1}{-\square+\rho^{2} / a^{2}}$,

where $\rho$ is a dimensionless parameter. The irrelevance by power counting of $S_{C}$ requires that in the continuum limit $\rho$ do not to vanish and $k_{\pi}$, as well as the product $k_{\pi} \rho$, do not diverge. Under these conditions, as a consequence of the explicit dependence on the lattice spacing of $C$ and the composites, operators of dimension higher than 4 are accompanied by the appropriate powers of the cut-off. All these parameters are obviously subject to renormalization.

We replace the chiral composites by the auxiliary fields

$\Sigma=\Sigma_{0}-i \gamma_{5} \vec{\tau} \cdot \vec{\Sigma}$

by means of the Stratonovich-Hubbard transformation 7 . Ignoring, as we will systematically do in the sequel, field independent factors, the partition function can be written

$$
\begin{aligned}
Z= & \int[d V]\left[\frac{d \Sigma}{\sqrt{2 \pi}}\right] \exp \left[-S_{Y M}-S_{0}\right] \\
& \int[d \bar{\lambda} d \lambda] \exp [(\bar{\lambda},(D-Q) \lambda)] \\
= & \int[d V]\left[\frac{d \Sigma}{\sqrt{2 \pi}}\right] \exp \left[-S_{Y M}-\tilde{S}\right]
\end{aligned}
$$

with

$\tilde{S}=S_{0}-\operatorname{Tr} \ln (D-Q)$

"Tr" is the trace over both space and internal degrees of freedom and we introduced the functions of the auxiliary fields

$$
\begin{aligned}
S_{0} & =-\frac{1}{4} \rho^{4}\left\langle\left(\Sigma^{\dagger},\left(a^{4} C\right)^{-1} \Sigma\right)\right\rangle, \\
D & =k_{\pi}\left[\rho^{2} \Sigma-m_{q}\right] .
\end{aligned}
$$

The pion field can be introduced by the transformation

$$
\Sigma=R\left[\frac{1-\gamma_{5}}{2} U+\frac{1+\gamma_{5}}{2} U^{\dagger}\right]
$$




$$
\begin{aligned}
R^{2} & =\Sigma_{0}^{2}+\vec{\Sigma}^{2} \\
U & =\exp \left(\frac{i}{f_{\pi}} \vec{\tau} \cdot \vec{\pi}\right) \in S U(2) .
\end{aligned}
$$

The volume element

$$
\left[\frac{d \Sigma}{\sqrt{2 \pi}}\right]=\left[\frac{d R}{\sqrt{2 \pi}}\right][d U] \exp \sum_{x} 3 \ln R
$$

provides the Haar measure $[d U]$ over the group. The effective action takes the form

$$
\begin{aligned}
\tilde{S}= & \sum_{\mu} \frac{1}{4}\left\langle\left(\nabla_{\mu}^{+}\left(R U^{\dagger}\right), \nabla_{\mu}^{+}(R U)\right)\right\rangle \\
& +\frac{\rho^{2}}{2 a^{2}}(R, R)-\operatorname{Tr} \ln (D-Q) .
\end{aligned}
$$

If the radial field $R$ acquires a nonvanishing expactation value $\bar{R}$, we set

$\bar{R}=f_{\pi}$

so that the first term of $\tilde{S}$ is the kinetic part of the chiral action while the radial field should not be dynamical because of its divergent mass (in the continuum limit). It can then be shown that the fermionic determinant can be expanded in powers of the derivatives of $U$, as appropriate to a Goldstone field, and of the explicit chiral symmetry breaking terms.

If we could naively forget about the Wilson term in the lattice action of the fermions, it would be very easy to evaluate

$\bar{R}=\sqrt{\frac{\Omega}{a \rho}}=f_{\pi}$,

where $\Omega=24$ is the number of quark components. If we neglect the fluctuations of $R$ and we put

$B=\frac{1}{2 a^{2} f_{\pi}}$,

the first term of $\tilde{S}$ can be identified with the leading term of the chiral models

$\mathcal{L}_{2}=\frac{1}{4} f_{\pi}^{2}\left[\left\langle\nabla_{\mu} U^{\dagger} \nabla^{\mu} U\right\rangle-2 m_{q} B\left\langle U+U^{\dagger}\right\rangle\right]$

and

$$
\begin{aligned}
m_{\pi}^{2} & =2 m_{q} B \\
k_{\pi}\langle 0|\bar{\lambda} \lambda| 0\rangle & =-2 f_{\pi}^{2} B
\end{aligned}
$$

while the fermionic determinant has an (hopping) expansion in inverse powers of $f_{\pi}$ and $k_{\pi}$ which provides corrections to $\mathcal{L}_{2}$. Furthermore the quarks are perturbatively confined in this vacuum because their effective mass

$$
M_{q}=m_{q}-k_{\pi} \rho^{2} \bar{R}=m_{q}-k_{\pi} \rho^{2} f_{\pi}
$$

is proportional to the inverse of the expansion parameters.

But since, as it is well known, in the absence of the Wilson term the lattice action is describing 16 fermionic species which cancel the abelian anomaly, let us go back to the full theory in the presence of the term $Q_{W}$. Let us set $U=U^{\dagger}=1$ and $R=\bar{R}$ and study the classical potential

$\mathcal{L}[\bar{R}]=\frac{1}{2} \frac{\rho^{2}}{a^{2}} \bar{R}^{2}-\frac{1}{2 a^{4} N^{4}} \operatorname{Tr} \ln P$

where $a^{4} N^{4}$ is the lattice volume

$$
P=M_{q}^{2}-2 M_{q} Q_{W}+Q_{W}^{2}-Q_{D}^{2}-\left[Q_{D}, Q_{W}\right]
$$

and the effective quark mass $M_{q}$ has been given above.

$\mathcal{L}[\bar{R}]$ is still a function of the fluctuating gauge fields. The variation of the partition function with respect to $\bar{R}$ yields the stationarity equations

$\frac{\rho^{2}}{a^{2}} \bar{R}=\frac{k_{\pi} \rho^{2}}{a^{4} N^{4}}\left\langle\left\langle\operatorname{Tr} P^{-1}\left(Q_{W}-M_{q}\right)\right\rangle\right\rangle$

where $\langle\langle\cdot\rangle\rangle$ is the functional average over the Yang Mills measure. Whenever a nonvanishing solution to these equations exists in the limit of vanishing of the explicit breaking terms, we have a spontaneous breaking of the chiral symmetry in $Q C D$.

Such solution must satisfy the condition on the effective quark mass $M_{Q}$

$\lim _{a \rightarrow 0} \frac{a M_{q}}{r}=0$.

This is the standard condition, necessary also with our effective action [11, for the chiral anomaly to be correctly reproduced.

The pion mass turns out to be

$m_{\pi}^{2}=\frac{k_{\pi} \rho^{2} \bar{R}}{f_{\pi}^{2}} \frac{1}{a^{4} N^{4}}\left\langle\left\langle\operatorname{Tr} P^{-1}\left(Q_{W}-m_{q}\right)\right\rangle\right\rangle$ 
and it is entirely due to the explicit breakings of the chiral invariance, namely the quark mass term and the Wilson term. It is convenient to introduce a subtracted mass which makes explicit the chiral limit

$m_{q}=m+\delta m$

with

$\delta m\left\langle\left\langle\operatorname{Tr} P^{-1}\right\rangle\right\rangle=\left\langle\left\langle\operatorname{Tr} P^{-1} Q_{W}\right\rangle\right\rangle$,

so that

$m_{\pi}^{2}=-m \frac{k_{\pi} \rho^{2} \bar{R}}{f_{\pi}^{2}} \frac{1}{a^{4} N^{4}}\left\langle\left\langle\operatorname{Tr} P^{-1}\right\rangle\right\rangle$.

Since the quark condensate in the present case is given by

$\langle 0|\bar{\lambda} \lambda| 0\rangle=\frac{1}{a^{4} N^{4}}\left\langle\left\langle\operatorname{Tr} P^{-1}\left(k_{\pi} \rho^{2} \bar{R}-m\right)\right\rangle\right\rangle$,

we get the Gell-Mann-Oakes-Renner relation [12]

$m_{\pi}^{2}=-\frac{1}{f_{\pi}^{2}} m\langle 0|\bar{\lambda} \lambda| 0\rangle$.

Eq. (33) shows a contribution to the effective quark mass proportional to the quark-condensate, that has therefore the same origin of the nonperturbative contribution to the quark mass first studied by Politzer [13] (see also [14).

In conclusion, with our choice of the irrelevant 4 -fermion $S_{C}$ we get the desired link between QCD and the chiral lagrangians, provided there exists a nontrivial solution for the expectation value of the radial field $R$ which is related to the spontaneous symmetry breaking of chiral invariance. It is indeed easy to show that the fermionic determinant can be expanded in powers of the derivatives of the auxiliary fields and the explicit chiral symmetry breaking terms. To complete the derivation of the chiral models from QCD it remains to show that these latter will generate only interactions of the chiral theories. This requires an investigation of the the additive renormalizations which are induced by the Wilson term $Q_{W}$ through the appropriate Ward identities. It could be of interest, also in this context, the use of Ginsparg-Wilson lattice fermions [15] in order to preserve as much as possible the explicit chiral invariance.
From a numerical point of view, the evaluation of the fermionic determinant should be faster in presence of the auxiliary fields, as they already provide the propagation of the pions. Some support to the above can be found in [16].

\section{REFERENCES}

1. S. Weinberg, The quantum theory of fields, Cambridge University Press 1996, Vol. II

2. J. Gasser and H. Leutwyler, Ann. Phys. 158 (1984) 142 and Nucl. Phys. B250 (1985) 465

3. K. G. Wilson, Phys. Rev. D10 (1974) 2445; and in "New phenomena in subnuclear physics", ed. A. Zichichi (Plenum, New York, 1977), Erice 1975

4. H. B. Nielsen and M. Ninomiya, Nucl. Phys. B185 (1981) 20

5. G. De Franceschi and F. Palumbo, Phys. Rev. D60 (1999) 074008, hep-th/9810218; F. Palumbo, Phys. Rev. D60 (1999) 074009, hep-lat/9905013.

6. S. Caracciolo and F. Palumbo, Nucl. Phys. B512 (1998) 505, hep-ph/9801389; Erratum, B555 (1999) 656

7. R. L. Stratonovitch, Dokl. Acad. Nauk. S.S.S.R. 115 (1957) 1097 [Sov. Phys. Dokl. 2 (1958) 416 ]; J. Hubbard, Phys. Rev. Lett. 3 (1959) 77

8. Y. Nambu and G. Jona-Lasinio, Phys. Rev. 122 (1961) 345.

9. D. J. Gross and A. Neveu, Phys. Rev. D10 (1974) 3235.

10. J. B. Kogut and D. K. Sinclair, Nucl. Phys. Proc. Suppl. 53 (1997) 272, hep-lat/9607083

11. S. Caracciolo and F. Palumbo, heplat/0004019

12. M. Gell-Mann, R. J. Oakes and B. Renner, Phys. Rev. 175 (1968) 2195

13. H. D. Politzer, Nucl. Phys. B117 (1976) 397

14. P. Pascual and E. de Rafael, Z. Phys. C 12 (1982) 127

15. P. H. Ginsparg and K. G. Wilson, Phys. Rev. D25 (1982) 2649.

16. R. C. Brower, K. Orginos and C. I. Tan, Nucl. Phys. Proc. Suppl. 42 (1995) 42, heplat/9501026 